\title{
Stage III High Grade Burkitt-Like Lymphoma
}

National Cancer Institute

\section{Source}

National Cancer Institute. Stage III High Grade Burkitt-Like Lymphoma. NCI Thesaurus.

Code C5000.

Stage III NHL means involvement of lymph node regions on both sides of the diaphragm

(III) that may also be accompanied by localized involvement of an extralymphatic organ or site (IIIE), by involvement of the spleen (IIIS), or both (IIIS+E). (from PDQ) 